\title{
Protein Kinase A governs growth and virulence in Candida tropicalis
}

\author{
Chi-Jan Lin, Chia-Yen Wu, Shang-Jie Yu, and Ying-Lien Chen \\ Department of Plant Pathology and Microbiology, National Taiwan University, 10617 Taipei, Taiwan
}

Candida tropicalis is one of the most important human fungal pathogens causing superficial infections in locations such as the oral mucosa and genital tract, as well as systemic infections with high mortality. In its sister species Candida albicans, the cyclic AMP/protein kinase A (cAMP/PKA) pathway regulates fungal adhesion and dimorphism, both of which correlate closely with virulence. CaTpk1 and CaTpk2, the catalytic subunits of PKA, not only share redundant functions in hyphal growth, adhesion, and biofilm formation, but also have distinct roles in stress responses and pathogenesis, respectively. However, studies on PKA in the emerging fungal pathogen $C$. tropicalis are limited. Our results suggest that Tpk1 is involved in cell wall integrity and drug tolerance. The tpk2/tpk2 mutants, which have no protein kinase $A$ activity, have reduced hyphal growth and adhesion. In addition, the tpk1/tpk1 tpk2/tpk2 double deletion mutant demonstrated delayed growth and impaired hyphal formation. In a murine model of systemic infection, both TPK1 and TPK2 were required for full virulence. We further found that EFG1 and HWP1 expression is regulated by PKA, while BCR1, FLO8, GAL4, and RIM101 are upregulated in the tpk1/tpk1 tpk2/tpk2 mutant. This study demonstrates that Tpk1 is involved in drug tolerance and cell wall integrity, while Tpk2 serves as a key regulator in dimorphism and adhesion. Both Tpk1 and Tpk2 are required for growth and full virulence in C. tropicalis.

1. Tpk1 and Tpk2 play a major role in growth

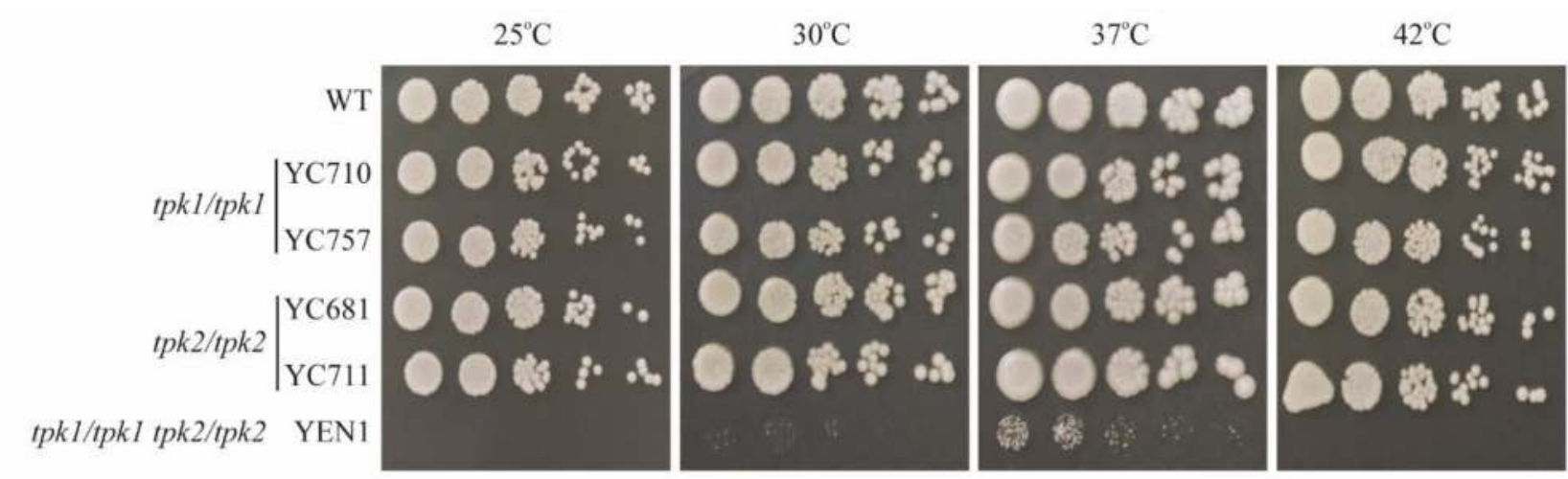

2. Tpk1 controls stress responses and drug tolerance

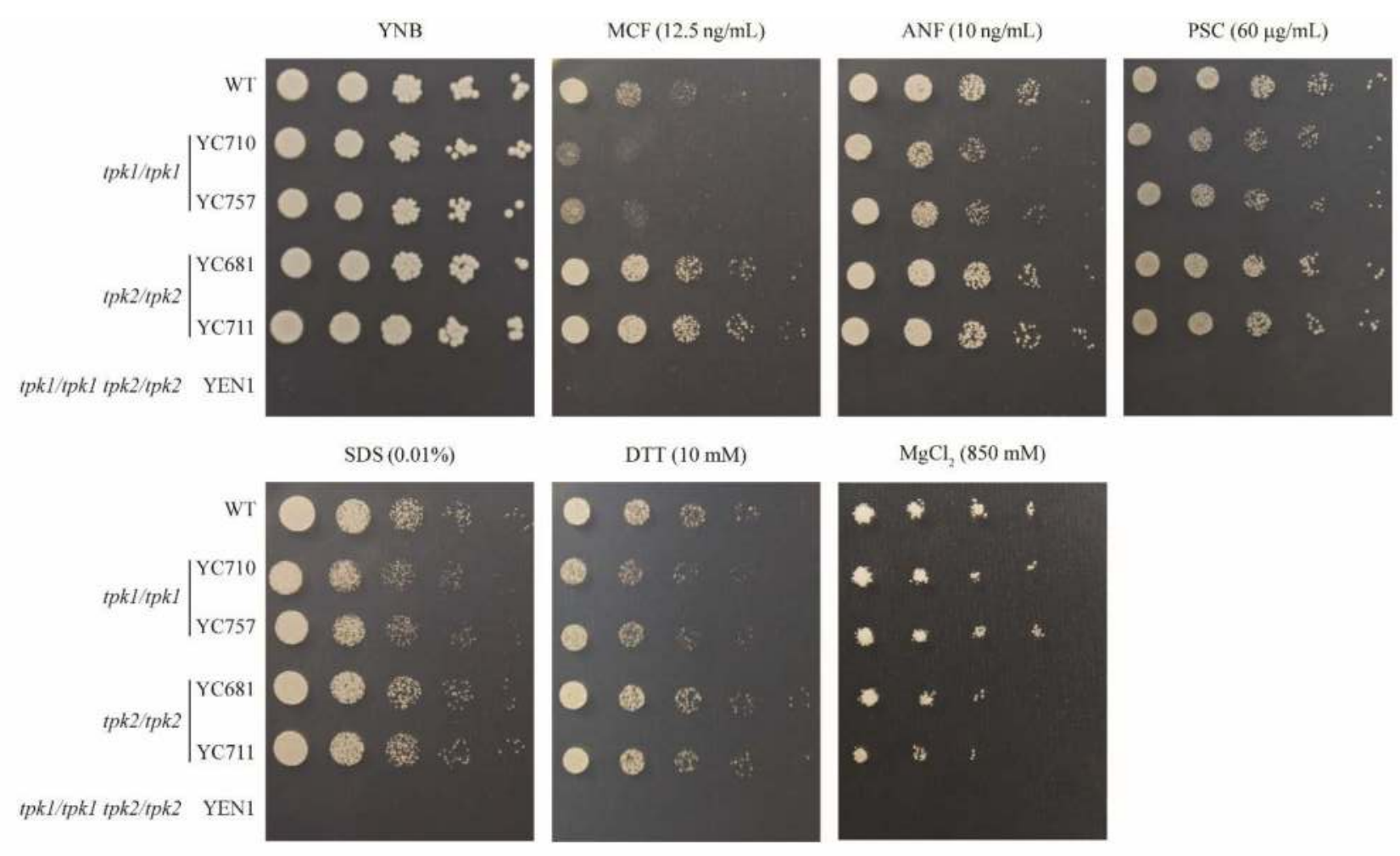

3. Tpk2 is involved in hyphal growth

A

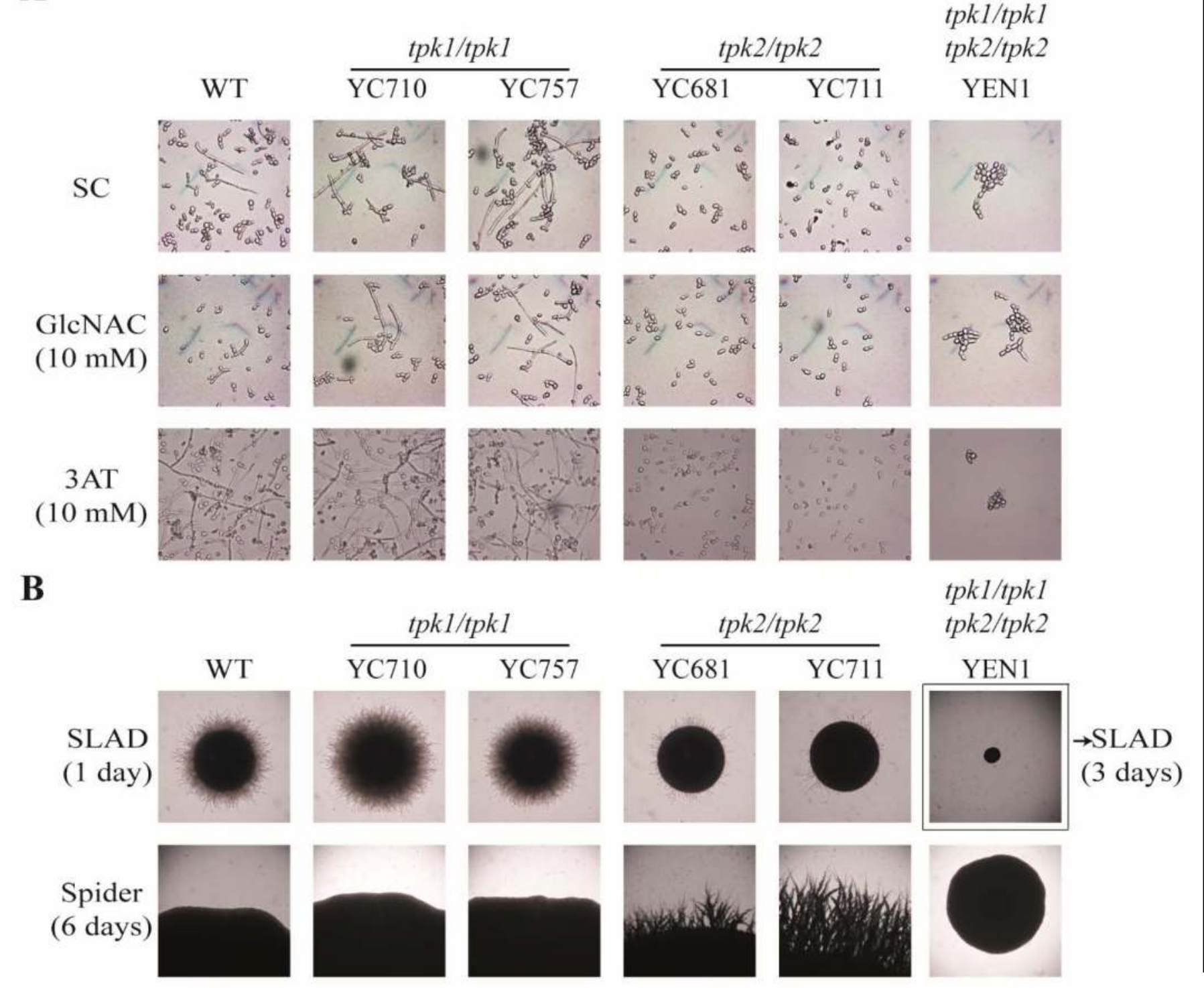

\section{Tpk2 regulates biofilm formation}

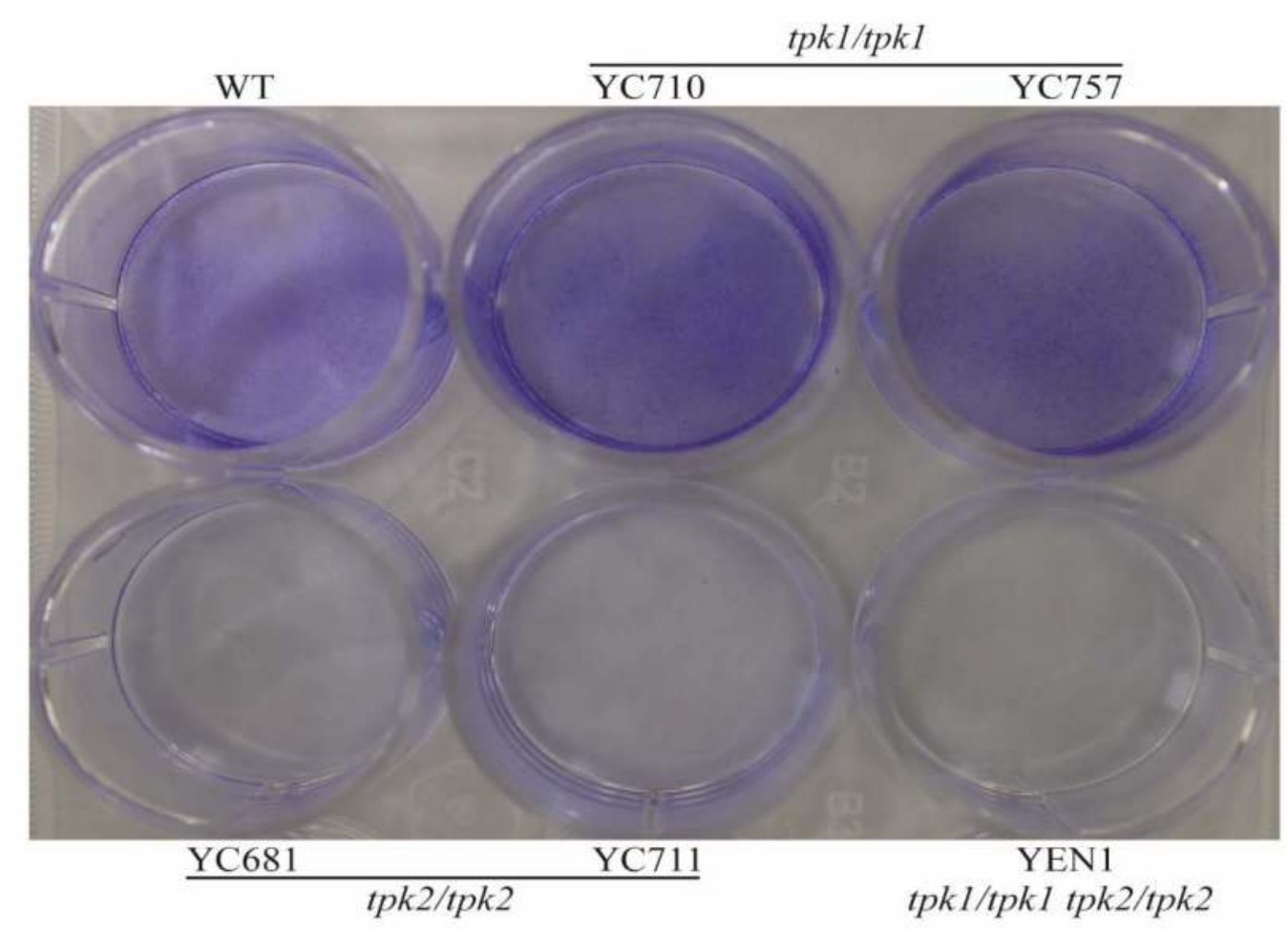

5. PKA null mutant is avirulent
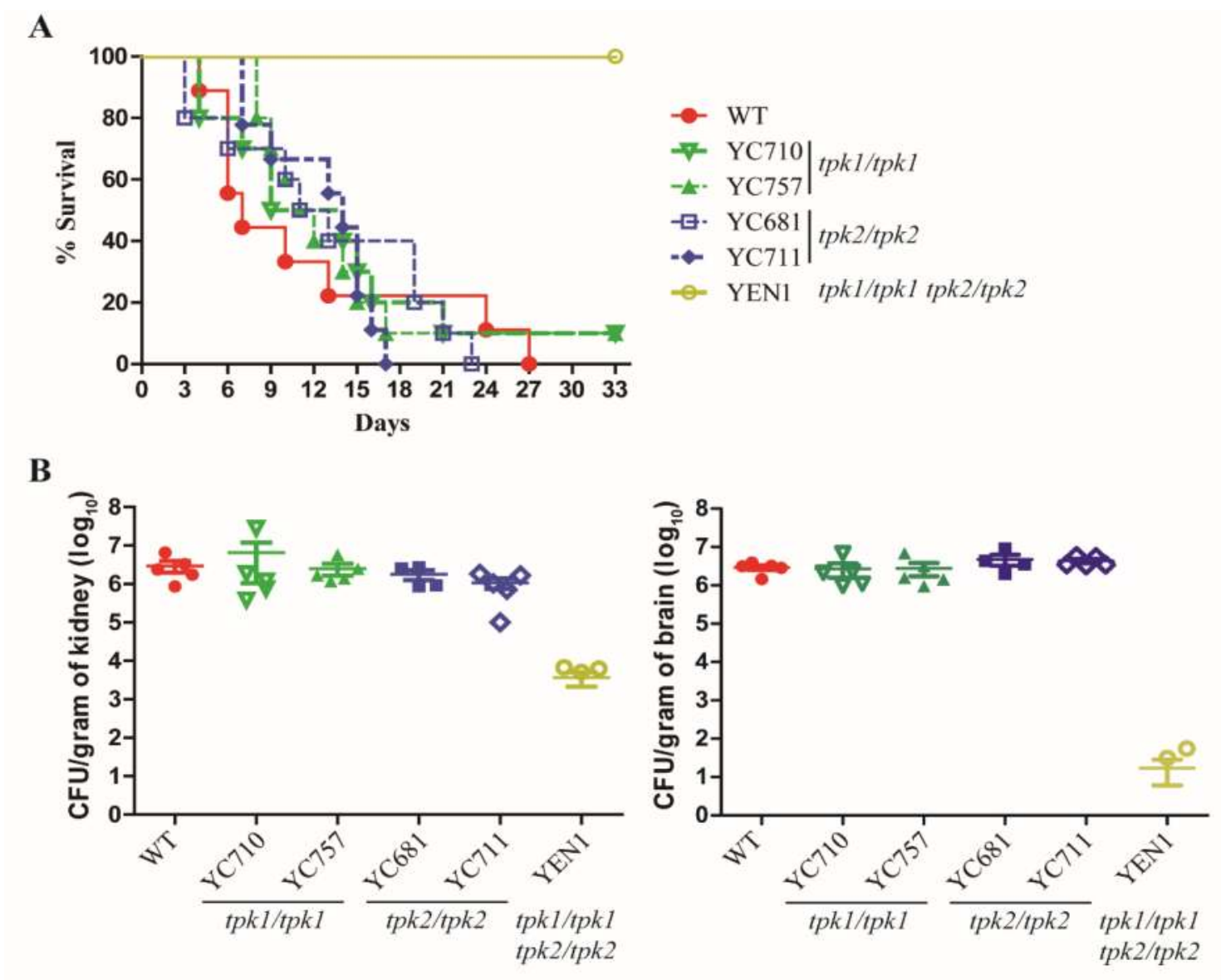

Model of PKA functions in C. tropicalis

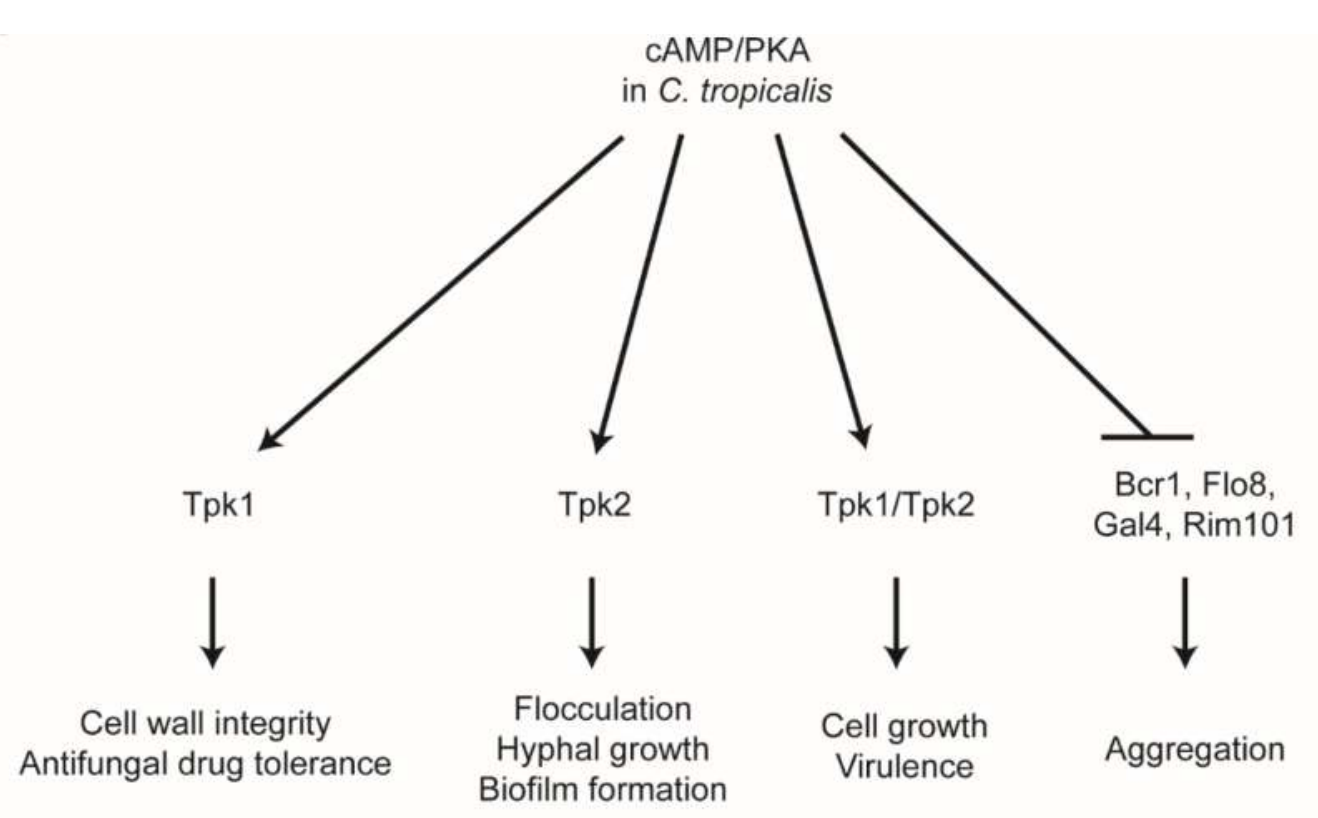

